\title{
Performance limits of channel parameter estimation for joint communication and positioning
}

\author{
Kathrin Schmeink ${ }^{*}$, Rebecca Adam and Peter A Hoeher
}

\begin{abstract}
Recently, a system concept for joint communication and positioning has been proposed by the authors. Channel parameter estimation (CPE) is the core part of this system proposal. Parameters of the physical channel, which can be exploited for positioning, are estimated based on the assumption that a priori knowledge about pulse shaping and receive filtering is available. At the same time, channel estimates of the equivalent discrete-time channel model, which are needed for data detection, are obtained inherently. This article focusses on the positioning part of the system proposal. Performance limits for CPE in terms of Cramer-Rao lower bounds are determined for different channel models. The influence of oversampling and of different channel characteristics is investigated. Oversampling proves especially helpful in dense multipath scenarios, which are most challenging. Based on the presented results, oversampling with a factor of two is recommended in order to improve the positioning accuracy. Excessive oversampling like in conventional global positioning system receivers is not necessary.
\end{abstract}

\section{Introduction}

Interest in joint communication and positioning is steadily increasing [1-4]. The combination of communication and positioning offers a wide range of advantages and synergistic effects like enhanced resource allocation or improved power control in cellular networks. Furthermore, applications such as locating emergency calls, tracking, and guiding firefighters or policemen on a mission, or location-based services become feasible. Communication and positioning can be combined in different ways: existing systems can be combined in a hybrid receiver, existing systems can be extended to provide additional services, or new systems with a unified signal structure can be designed. The latter approach is considered in this article. The main aim of such a joint communication and positioning system is to provide high data rates with low bit error rate for the communication part, and a high localization accuracy for the positioning part. Since it is quite challenging to fulfil both conditions at the same time, a flexible system configuration is desirable in order

*Correspondence: kas@tf.uni-kiel.de

Information and Coding Theory, Faculty of Engineering, University of Kiel, Kaiserstrasse 2, 24143 Kiel, Germany adjust the tradeoff between communication and positioning to the recent needs: in case of an emergency call, emphasis can be laid on the positioning part, whereas the communication payload can be increased in case of data transmissions like file downloads or video streaming. Furthermore, a flexible configuration allows adapting the transmission scheme to changing channel conditions in order to fulfil a certain quality of service.

Recently, a system concept for joint communication and positioning has been proposed by the authors of $[5,6]$. The system proposal is based on multi-layer interleavedivision multiple access (ML-IDMA) [7] in combination with pilot layer-aided channel estimation (PLACE) [8]. ML-IDMA is a combination of interleave-division multiplexing (IDM) and IDMA. The application of IDM as multiplexing scheme is essential for the system proposal since it provides the desired flexibility, while the choice of the multiple access scheme is less crucial. IDM is a special code-division multiplexing scheme, where different data streams (called layers) are separated by layer-wise interleaving. The key idea for joint communication and positioning is to allocate one layer with a known training pattern employed for positioning and channel estimation, whereas the remaining layers are information-bearing 
layers employed for data communication. This article focusses on the positioning part of the proposed system, which is based on the time-of-arrival (ToA) concept: Radiolocation is typically performed in two steps [9]. First, parameters of the physical channel like the received signal strength, the angle-of-arrival, or the ToA are estimated (parameter estimation). Based on these parameters, the position of the mobile station is determined in a second step (position estimation). The parameter estimation error translates into a positioning error via the geometric dilution of precision (GDOP) [10]. Given a certain GDOP, the positioning accuracy increases if the parameter estimation error decreases. Thus, accurate parameter estimation is a prerequisite for precise radiolocation.

Channel parameter estimation (CPE) is the core part of the proposed system concept. Based on the assumption that a priori information about pulse shaping and receive filtering is available, parameters of the physical channel including the ToA are estimated and exploited for positioning. At the same time, estimates for the channel coefficients of the equivalent discrete-time channel model, which are needed for data detection, are obtained inherently. Thus, CPE enables positioning and data detection. Typically, only one aspect of CPE is considered: On the one hand, CPE is well known in the context of channel sounding [11-13]. In this case, the parameters of the physical channel are of interest, while the channel coefficients of the equivalent discrete-time channel model, which are available as well, are not further processed. On the other hand, the usage of a priori information about pulse shaping and receive filtering has already been suggested in [14-16] for improved channel estimation in communication systems. In this case, the information about the physical channel is discarded. For joint communication and positioning, both aspects of CPE are exploited.

The channel parameter estimator considered in this contribution is based on the maximum-likelihood principle. There are two equivalent approaches: On the one hand, the channel parameters can be estimated directly from the received samples as it is often the case for channel sounding [11-13]. Similar estimators have been investigated for example in the context of Rake receivers in code-division multiple access [17] or for pure navigation purposes [18]. On the other hand, CPE can be performed in two steps: First, standard channel estimation (without a priori information about pulse shaping and receive filtering) is applied in order to obtain a preliminary estimate of the channel coefficients. Based on this pre-stage channel estimates, the parameters of the physical channel are estimated and enhanced channel estimates are obtained. This two-step approach is for example considered in [19]. Similar to the channel estimation approaches in [14-16], the channel estimates which are available after CPE are more reliable than the pre-stage channel estimates due to the exploitation of $a$ priori information about pulse shaping and receive filtering. Since the approach based on the received samples and the two-step approach based on the pre-stage channel estimates are equivalent, the latter approach is recommended due to complexity reasons.

In this article, performance limits for parameter estimation in terms of Cramer-Rao lower bounds (CRLBs) are determined for different channel models. Especially, the impact of oversampling is analyzed. Three different kinds of channel models are considered: A single-path channel, several two-path channels, and different wireless world initiative new radio (WINNER) channels with numerous multipath components. The single-path channel is taken into account since it is the best possible case for positioning and, thus, provides a lower bound for all other channel models. By means of the two-path channel models, the influence of different channel characteristics such as the excess delay, power ratio, and phase offset of the propagation paths can be investigated. The results obtained for the two-path channels are the basis for more realistic channel models with an arbitrary number of propagation paths like the WINNER channel models that are considered in this article. It is observed that oversampling provides a performance gain compared to symbol-rate sampling. Oversampling proves especially helpful in dense multipath scenarios, which are most challenging with respect to positioning. Since the performance for all oversampling factors larger than two is about the same, oversampling with a factor of two is recommended.

Many applications of joint communication and positioning are located in urban or indoor areas including hotspots like train stations, airports, or shopping malls. In these environments, multipath components are typically dense, i.e., these environments are very demanding concerning radiolocation. However, the required positioning accuracy is quite high in urban or indoor areas. Often, it is not possible to meet the required accuracy with a single radiolocation method. Therefore, several radiolocation methods should be combined via sensor fusion [20-22] in order to improve the positioning accuracy. Keeping in mind that a system can always be extended by assisting concepts, the system proposal should be understood as a single contribution concerning positioning, that can be combined with other radiolocation methods via sensor fusion.

The remainder of this article is organized as follows: In Section "System concept", the system and channel model is introduced and the joint communication and positioning system proposed by the authors is presented. CPE is explained in Section "CPE". The basic estimation problem is introduced and the twostep maximum-likelihood approach is presented. In Section "Performance limits-CRLB", performance limits for the derived channel parameter estimator are 
determined in terms of CRLBs. Numerical results for different channel models and for different oversampling factors are presented. Furthermore, the impact of the obtained results on the overall positioning process are discussed. Finally, conclusions are drawn in Section "Conclusion".

\section{System concept}

Throughout this article, the discrete-time complex baseband notation is used. Let $x^{\prime}[\kappa], 0 \leq \kappa<K^{\prime}$, denote the $\kappa$ th symbol of a coded and modulated burst of length $K^{\prime}$. If oversampling is applied, this sequence is upsampled to a burst of length $K=J K^{\prime}$, where $J$ is the oversampling factor. The symbols of the upsampled sequence are given according to

$$
x[k]= \begin{cases}x^{\prime}[k / J] & \text { if } k \bmod J=0, \\ 0 & \text { else. }\end{cases}
$$

In case of symbol-rate sampling $(J=1)$, both sequences are the same $\left(x[k]=x^{\prime}[\kappa]\right.$ and $\left.K=K^{\prime}\right)$. Assuming a linear modulation scheme, the received sample $y[k]$ at time index $k$ is given by

$$
y[k]=\sum_{l=0}^{L} h_{l}[k] \cdot x[k-l]+n[k], 0 \leq k<K+L,
$$

where $h_{l}[k]$ is the $l$ th channel coefficient of the equivalent discrete-time channel model with channel memory length $L$ and $n[k]$ is a zero mean Gaussian noise sample. The equivalent discrete-time channel model comprises all continuous-time elements of a transmission link, namely the pulse shaping filter $g_{\mathrm{Tx}}(\tau)$, the time-variant physical channel $c(\tau, t)$, additive white Gaussian noise (AWGN), the receive filter $g_{R x}(\tau)$, and sampling. This means that the channel coefficients $h_{l}[k]$ are the samples of the overall channel weight function $h(\tau, t)$, which is given by the convolution of $g_{\mathrm{Tx}}(\tau), c(\tau, t)$, and $g_{\mathrm{Rx}}(\tau)$. Due to the associative and commutative properties of the convolution, pulse shaping and receive filtering can be combined: $g(\tau)=g_{\mathrm{Tx}}(\tau) * g_{\mathrm{Rx}}(\tau)$. The physical channel $c(\tau, t)$ is typically modeled by a weighted sum of delayed Dirac impulses. In this case, the channel coefficients after sampling at $t=k \mathcal{T}+\varepsilon$ are given by

$$
h_{l}[k]=\sum_{i=1}^{I} f_{i}[k] \cdot g\left(l \mathcal{T}+\varepsilon-\tau_{i}[k]\right) .
$$

where $f_{i}[k] \in \mathbb{C}$ and $\tau_{i}[k] \in \mathbb{R}_{\geq 0}$ are the complex amplitude and the propagation delay of the $i$ th propagation path, respectively. Furthermore, $I$ denotes the number of propagation paths, $\mathcal{T}=T_{s} / J$ denotes the sampling period, which is given as a fraction of the symbol duration $T_{s}$, and $\varepsilon$ is the sampling phase, that accounts for sampling time offsets. The noise process $n[k]$ in (2) is generally colored because white Gaussian noise with zero mean and variance $\sigma_{n}^{2}$ is added to the continuous-time signal before receive filtering, i.e., the white Gaussian noise is filtered by $g_{\mathrm{Rx}}(\tau)$. Thus, the sampled autocorrelation function of $n[k]$ is given by

$$
\varphi_{n n}[\Delta k]=\sigma_{n}^{2} \cdot \psi_{\mathrm{Rx}}(\Delta k \mathcal{T})
$$

with $\Delta k=k_{1}-k_{2}$ and where $\psi_{\mathrm{Rx}}(\tau)=g_{\mathrm{Rx}}(\tau) * g_{\mathrm{Rx}}(-\tau)$ denotes the autocorrelation function of the receive filter. If a square-root Nyquist pulse is applied at the receiver, the noise remains white for symbol-rate sampling.

The channel coefficients in (3) depend on propagation delays $\tau_{i}[k]$ of the physical channel. For positioning based on the ToA, the propagation delay of the first arriving path, $\tau_{1}[k]$, needs to be estimated. In contrast, perfect synchronization is often assumed for the simulation of communication systems. This means that the propagation delay of the first arriving path is known and eliminated perfectly such that excess delays $v_{i}[k]=\tau_{i}[k]-\tau_{1}[k]$ are considered only. The sampling phase $\varepsilon$ is zero in this case. Consequently, the leading channel coefficients with zero values are eliminated and a shorter channel memory length can be taken into account. The assumption of perfect synchronization is not applicable in this contribution since the positioning part of the proposed system concept is based on the ToA. Therefore, a coarse synchronization is considered subsequently, that eliminates the propagation delay of the first arriving path only approximately:

$$
v_{i}^{\prime}[k]=\tau_{i}[k]-\check{\tau}_{1}[k]=\tau_{i}[k]-\left(\tau_{1}[k]+\varepsilon\right)=v_{i}[k]-\varepsilon .
$$

This means that excess delays in combination with a nonzero sampling phase are taken into account. In this case, the propagation delays in (3) are replaced by excess delays:

$$
h_{l}[k]=\sum_{i=1}^{I} f_{i}[k] \cdot g\left(l \mathcal{T}+\varepsilon-v_{i}[k]\right) .
$$

Based on the above assumptions, the estimation of the ToA $\hat{\tau}_{1}[k]$ corresponds to the estimation of the sampling phase $\hat{\varepsilon}$. The final ToA estimate, that can be exploited for positioning, is given as

$$
\hat{\tau}_{1}[k]=\check{\tau}_{1}[k]-\hat{\varepsilon}=\tau_{1}[k]+\varepsilon-\hat{\varepsilon} .
$$

It should be noted here that the requirements concerning synchronization differ for communication and positioning purposes. Assuming that a correlation-based synchronization is performed, the highest correlation peak should be chosen for communication purposes in order to maximize the signal-to-noise ratio (SNR) at the receiver side. In contrast, the first correlation peak, which might not be the highest, is important for positioning via the ToA. With the proposed system concept both requirements can be met: 
First, a coarse synchronization is performed that maximizes the SNR. Then, the ToA is determined more accurately by estimating the sampling phase using CPE. Thus, $\mathrm{CPE}$ corresponds to a fine-tuning of synchronization for positioning purposes.

Now, it becomes clear why CPE is the core part of the proposed joint communication and positioning system. Channel estimation is mandatory for communication purposes since the channel coefficients of the equivalent discrete-time channel model need to be known for data detection. If the parameters of the physical channel are estimated, positioning is enabled and estimates of the channel coefficients are available inherently. The relationship in (6) is the basis for CPE. From (6), it is obvious that the channel coefficients are known if the parameters of the physical channel $\left(f_{i}[k], v_{i}[k], 1 \leq i \leq I\right)$ and the shape of the filter $g(\tau)$ are known. Training symbols should be inserted into the transmission burst in order to simplify CPE. All multiplexing techniques including time-division multiplexing (TDM) and frequency-division multiplexing can be applied for that purposes. According to the system proposal in [5,6], IDM [7] in combination with PLACE [8] is considered in this article. The main idea of IDM is to linearly superimpose several data streams of a user, which are called layers in the following. In case of PLACE, a pilot layer containing training symbols is additionally superimposed onto the data layers for CPE purposes as shown in Figure 1. Each data layer is either dedicated to communication purposes (e.g., speech or video transmission) or it may carry auxiliary information for localization purposes (e.g., time of departure or positions of reference objects). The layers are distinguished by layer-specific interleavers: Let $u_{m}[n], 0 \leq n<N, 1 \leq m \leq M$, denote the $n$th bit of the $m$ th data layer. Each bit sequence is encoded with code rate $R=N / K^{\prime}$ (ENC), interleaved by a layer-specific interleaver $\left(\pi_{m}\right)$ and mapped onto the complex plane via binary phase shift keying (BPSK), which leads to the layerwise symbols $x_{m}^{\prime}[\kappa]$. Before all data layers and the pilot layer with training symbols $x_{0}^{\prime}[\kappa]$ are summed up, an adequate power and phase allocation with complex weighting factors $a_{m} \mathrm{e}^{\mathrm{j} \xi_{m}}$ is performed. Thus, the $\kappa$ th symbol of the transmission burst of length $K^{\prime}$ is given by

$$
\begin{aligned}
x^{\prime}[\kappa] & =\sum_{m=0}^{M} a_{m} \mathrm{e}^{\mathrm{j} \xi_{m}} \cdot x_{m}^{\prime}[\kappa] \\
& =\underbrace{a_{0} \mathrm{e}^{\mathrm{j} \xi 0} \cdot x_{0}^{\prime}[\kappa]}_{\text {pilot layer }}+\underbrace{\sum_{m=1}^{M} a_{m} \mathrm{e}^{\mathrm{j} \xi_{m}} \cdot x_{m}^{\prime}[\kappa]}_{\text {data layers }} .
\end{aligned}
$$

Each symbol $x^{\prime}[\kappa]$ carries $\mathcal{B}=R M$ bits, where $\mathcal{B}$ is called bit load [7]. Since all layers employ the same encoders in combination with BPSK mapping, the transmitter structure of IDM is very simple. However, IDM offers a flexible configuration, which is desirable for joint communication and positioning, because the data rate can be easily adapted by changing the number of data layers $M$ instead of changing the modulation scheme [23]. Furthermore, layer-wise unequal error protection can easily be achieved by assigning different amplitude levels to different layers [7]. Similarly, the tradeoff between communication and positioning purposes can be regulated via an adequate power allocation. The ratio of the pilot layer power to the total power,

$$
\rho=\frac{a_{0}^{2}}{\sum_{m=0}^{M} a_{m}^{2}},
$$

can be varied between 0 and 1 , where $\rho=0$ and $\rho=1$ correspond to no training at all and pure training, respectively.

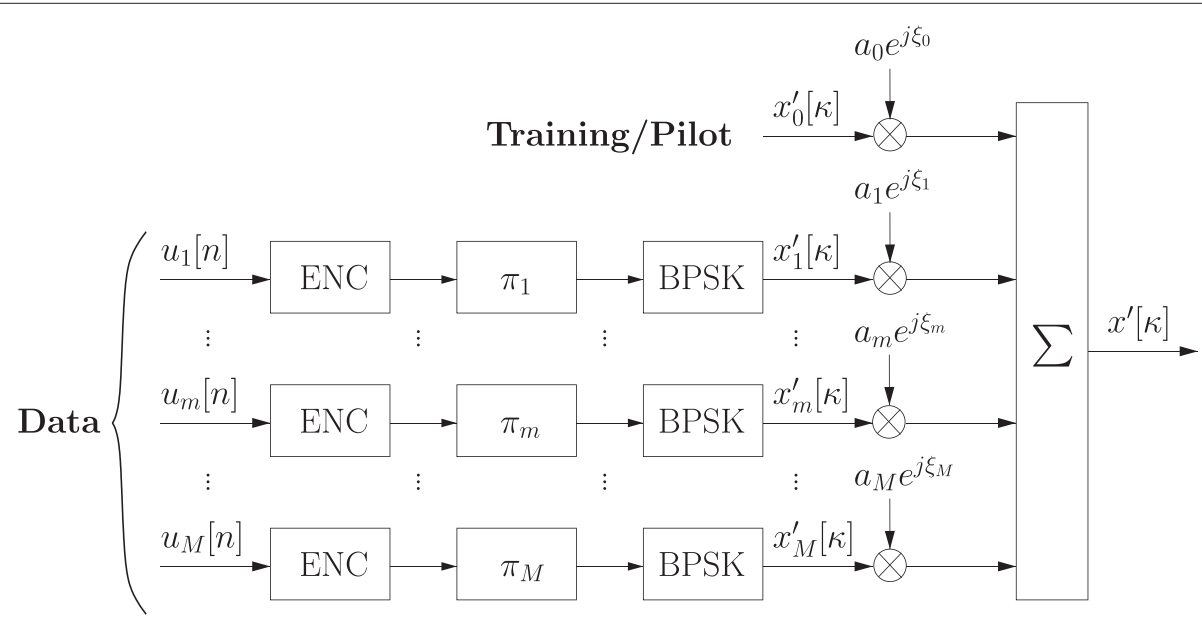

Figure 1 Transmitter structure of IDM with PLACE. 


\section{CPE}

For the purpose of CPE, the channel model in (6) is reformulated by combining the excess delays with the sampling phase to auxiliary parameters $\varrho_{i}[k]=v_{i}[k]-\varepsilon, 1 \leq i \leq I$, which are termed coarse excess delays in the following. Furthermore, block fading is assumed, i.e., the parameters of the physical channel do not change over the transmission burst. In this case, the channel coefficients do not depend on the time index $k$ anymore:

$$
h_{l}=\sum_{i=1}^{I} f_{i} \cdot g\left(l \mathcal{T}-\varrho_{i}\right) .
$$

Block fading can be assumed for $0 \leq B_{D} \cdot K^{\prime} T_{s}<0.01$, where $B_{D}$ is the Doppler spread of the physical channel, $K^{\prime}$ is the burst length and $T_{s}$ is the symbol duration. The Doppler spread depends on the mobility of the investigated scenario and can be equated with the maximum Doppler shift $B_{D}=f_{D, \max }=v / c \cdot f_{0}$. In this case, $v$ represents the maximum possible velocity, $c$ denotes the speed of light, and $f_{0}$ is the carrier frequency. For joint communication and positioning, mainly indoor and urban areas are of interest. Hence, the maximum velocities, that typically occur, lie between 7 and $70 \mathrm{~km} / \mathrm{h}$. Thus, a wide range of reasonable parameter combinations $\left(K^{\prime}, T_{s}, f_{0}\right)$ exists, for which the block fading assumption is valid. If the parameters are fixed to specific values, that can not be changed, and the block fading assumption is violated, the transmission burst can be subdivided into smaller blocks, for which the block fading assumption is valid again. In this case, CPE can be performed block-instead of burst-wise (sliding window approach, see also [8]). Consequently, the assumption of block fading is adequate and hardly restricts the applicability of the proposed channel parameter estimator.

In order to emphasize the functional relationship between the parameters of the physical channel $\left(f_{i}, \varrho_{i}, 1 \leq\right.$ $i \leq I)$ and the channel coefficients of the equivalent discrete-time channel $h_{l}$, the channel parameters are stacked in a vector

$$
\begin{aligned}
\boldsymbol{\theta} & =\left[\theta_{1}, \ldots, \theta_{P}\right]^{T} \\
& =\left[\operatorname{Re}\left\{f_{1}\right\}, \operatorname{Im}\left\{f_{1}\right\}, \varrho_{1}, \cdots, \operatorname{Re}\left\{f_{I}\right\}, \operatorname{Im}\left\{f_{I}\right\}, \varrho_{I}\right]^{T}
\end{aligned}
$$

of length $P=3 I$. Each propagation path is characterized by three parameters: the real and imaginary part of the complex amplitude, $\operatorname{Re}\left\{f_{i}\right\}$ and $\operatorname{Im}\left\{f_{i}\right\}$, and the coarse excess delay $\varrho_{i}$. The channel coefficients in (10) can be expressed as a function of the parameter vector in (11) according to

$$
h_{l}(\boldsymbol{\theta})=\sum_{\substack{p=1 \\ p+=3}}^{P}\left(\theta_{p}+j \theta_{p+1}\right) g\left(l \mathcal{T}-\theta_{p+2}\right) .
$$

Based on the training symbols $x_{0}[k], \mathrm{CPE}$ is performed: Inserting (8) into (2) leads to:

$$
\begin{aligned}
y[k]= & \sum_{l=0}^{L} \sum_{m=0}^{M} h_{l}(\boldsymbol{\theta}) \cdot a_{m} \mathrm{e}^{\mathrm{j} \xi_{m}} \cdot x_{m}[k-l]+n[k] \\
= & \underbrace{\sum_{l=0}^{L} h_{l}(\boldsymbol{\theta}) \cdot a_{0} \mathrm{e}^{\mathrm{j} \xi 0} \cdot x_{0}[k-l]}_{\text {useful part for CPE }} \\
& +\underbrace{\sum_{l=0}^{L} \sum_{m=1}^{M} h_{l}(\boldsymbol{\theta}) \cdot a_{m} \mathrm{e}^{\mathrm{j} \xi_{m}} \cdot x_{m}[k-l]}_{\text {data layer interference }}+n[k] .
\end{aligned}
$$

Only the first part in (13) is useful for CPE, while the second part (data layer interference) complicates CPE. Typically, turbo-type iterative receivers are applied for data detection in case of IDM and related techniques [24-26], i.e., the receiver consists of a multi-layer detector (MLD) and a bank of layer-wise decoders. At the MLD, only the multiplexing and the channel constraint are taken into account ignoring the coding constraint. In contrast, the layer-wise decoders consider the coding constraint only. Extrinsic information is exchanged iteratively between the MLD and the decoders. In this way, the quality of the incorporated estimates can be increased over iterations. In case of channel estimation, the feedback information from the decoders can be used to mitigate the data layer interference [8]: By means of data layer interference cancellation (DIC), improved channel estimates are obtained, which in turn lead to improved data estimates. Typically, the data layer interference can be cancelled nearly perfectly, i.e., the residual data layer interference after an adequate number of receiver iterations is negligible. Under certain conditions, the residual data layer interference is not negligible anymore. In this case, the residual data layer interference can be modeled as a Gaussian variable according to the central limit theorem because $M$ and $L$ are typically large. Then, the noise and the residual data layer interference can easily be combined to a single Gaussian distortion with increased variance compared to pure noise, i.e., non-negligible residual data layer interference corresponds to a decrease in SNR. Hence, it is sufficient to consider perfect DIC for the derivation of performance limits for CPE. This means that CPE is first performed after an adequate number of receiver iterations. Previously, standard channel estimation is applied. The assumption of perfect DIC leads to

$$
y_{\text {DIC }}[k]=\sum_{l=0}^{L} h_{l}(\boldsymbol{\theta}) \cdot a_{0} \mathrm{e}^{\mathrm{j} \xi 0} \cdot x_{0}[k-l]+n[k] .
$$


Without loss of generality, $\xi_{0}=0$ can be assumed. The remaining phases $\xi_{m}$ are distributed equally between 0 and $\pi$. Furthermore, the amplitude of the pilot layer can be expressed as $a_{0}=\sqrt{\rho}$ due to power normalization $\left(\sum_{m=0}^{M} a_{m}^{2} \stackrel{!}{=} 1\right)$. Based on the observations after DIC in (14), the parameter vector $\boldsymbol{\theta}$ can be estimated via the maximum-likelihood $(M L)$ approach $[27,28]$ exploiting $a$ priori information about the pilot symbols $x_{0}[k]$ and the overall pulse shape $g(\tau)$ (including pulse shaping and receive filtering). ML estimators are asymptotically optimal (unbiased and efficient), i.e., they achieve the best possible performance given by the CRLB for a large number of observations or at high SNR. Furthermore, the ML estimators correspond to the least-squares (LS) estimators in case of Gaussian noise. For the derivation of the ML estimator for CPE, it is useful to express (14) in vector/matrix notation:

$$
\boldsymbol{y}_{\mathrm{DIC}}=\boldsymbol{X}_{0} \boldsymbol{h}(\boldsymbol{\theta})+\boldsymbol{n},
$$

where $\boldsymbol{y}_{\mathrm{DIC}}=\left[y_{\mathrm{DIC}}[L], \ldots, y_{\mathrm{DIC}}[K-1]\right]^{T}$ is the observation vector containing the received samples after DIC and $X_{0}$ is the training matrix with Toeplitz structure:

$$
\boldsymbol{X}_{0}=\sqrt{\rho} \cdot\left[\begin{array}{cccc}
x_{0}[L] & x_{0}[L-1] & \cdots & x_{0}[0] \\
x_{0}[L+1] & x_{0}[L] & \cdots & x_{0}[1] \\
\vdots & \vdots & \ddots & \vdots \\
x_{0}[K-1] & x_{0}[K-2] & \cdots & x_{0}[K-L-1]
\end{array}\right] \text {. }
$$

Furthermore, $\boldsymbol{h}(\boldsymbol{\theta})=\left[h_{0}(\boldsymbol{\theta}), \ldots, h_{L}(\boldsymbol{\theta})\right]^{T}$ and $\boldsymbol{n}=$ $[n[L], \ldots, n[k-1]]^{T}$ denote the channel coefficient vector and a zero mean Gaussian noise vector with covariance matrix $C_{n}$, respectively. The entries of the noise covariance matrix are determined according to the autocorrelation function of the noise process given in (4):

$$
\left[\boldsymbol{C}_{\boldsymbol{n}}\right]_{i, j}=\sigma_{n}^{2} \psi_{\mathrm{Rx}}((i-j) \mathcal{T})
$$

As already mentioned above, there are two equivalent approaches to estimate the channel parameters $\theta$ based on the signal model in (15)-(17). The first approach is based on the received samples $y_{\text {DIC }}$ and the second approach relies on so-called pre-stage channel estimates $\breve{h}$ that are obtained via a standard channel estimation algorithm from the received samples $\boldsymbol{y}_{\mathrm{DIC}}$. Due to complexity reasons, only the second approach is considered subsequently. Since block fading is assumed, the pre-stage channel estimates can be obtained in closed form via (weighted) least-squares channel estimation [28]:

$$
\begin{aligned}
\check{\boldsymbol{h}}= & \left(\boldsymbol{X}_{0}^{H} \boldsymbol{C}_{\boldsymbol{n}}^{-1} \boldsymbol{X}_{0}\right)^{-1} \boldsymbol{X}_{0}^{H} \boldsymbol{C}_{\boldsymbol{n}}^{-1} \boldsymbol{y}_{\mathrm{DIC}}, \\
= & \underbrace{\left(\boldsymbol{X}_{0}^{H} \boldsymbol{C}_{\boldsymbol{n}}^{-1} \boldsymbol{X}_{0}\right)^{-1} \boldsymbol{X}_{0}^{H} \boldsymbol{C}_{\boldsymbol{n}}^{-1} \boldsymbol{X}_{0}}_{\mathcal{I}} \boldsymbol{h}(\boldsymbol{\theta}) \\
& +\underbrace{\left(\boldsymbol{X}_{0}^{H} \boldsymbol{C}_{\boldsymbol{n}}^{-1} \boldsymbol{X}_{0}\right)^{-1} \boldsymbol{C}_{\boldsymbol{n}}^{-1} \boldsymbol{X}_{0}^{H} \boldsymbol{n}}_{\eta}
\end{aligned}
$$

where $\eta \sim \mathcal{C N}\left(\mathbf{0}, C_{\eta}\right)$ is the channel estimation error with covariance matrix

$$
\boldsymbol{C}_{\boldsymbol{\eta}}=\left(\boldsymbol{X}_{0}^{H} \boldsymbol{C}_{\boldsymbol{n}}^{-1} \boldsymbol{X}_{0}\right)^{-1} .
$$

At this stage, the a priori information about pulse shaping and receive filtering is not exploited yet. The $a$ priori information about pulse shaping and receive filtering is incorporated in a second step applying the ML principle: The parameters of the physical channel $\boldsymbol{\theta}$ are estimated by maximizing the likelihood function $p(\breve{\boldsymbol{h}}, \boldsymbol{\theta})[28]$ :

$$
\begin{aligned}
\hat{\boldsymbol{\theta}} & =\arg \max _{\tilde{\boldsymbol{\theta}}}\{p(\check{\boldsymbol{h}}, \tilde{\boldsymbol{\theta}})\} \\
& =\arg \min _{\tilde{\boldsymbol{\theta}}}\left\{(\check{\boldsymbol{h}}-\boldsymbol{h}(\tilde{\boldsymbol{\theta}}))^{H} \boldsymbol{C}_{\boldsymbol{\eta}}^{-1}(\check{\boldsymbol{h}}-\boldsymbol{h}(\tilde{\boldsymbol{\theta}}))\right\} .
\end{aligned}
$$

Due to the pulse shape $g(\tau)$, the metric in (20) is nonlinear, i.e., the minimization cannot be solved in closed form. Exhaustive search is prohibitive because the search space (parameter space) is continuous and of high dimension $(P=3 I)$. Hence, an optimization method needs to be applied, which typically performs the minimization/maximization in an iterative manner. Due to the superposition of several multipath components, there exist many local optima besides the global optimum. Hence, a global optimization method is required in general. A viable global optimization algorithm is for example particle swarm optimization (PSO) [29,30] as demonstrated by the authors in [31]. Of course, other optimization methods are applicable as well. If a priori information about the approximate location of the global optimum is available, even local optimization methods like the Levenberg-Marquardt algorithm [32, pp. 688-693] can be applied. For example, the parameter estimate of the preceding burst can be used as initial guess for the recent burst if the channel is changing slowly from burst to burst (tracking). However, a global optimization method like PSO is still required for acquisition. Even though the global optimization methods may be computationally complex in the acquisition phase, they can be reduced in complexity in the tracking phase, where real-time operation is more critical: The a priori information about the approximate location of the global optimum can be 
exploited by global optimization methods to narrow the search space and to simplify the global search. In this case, PSO needs much less computations on average compared to acquisition. In general, there is a tradeoff between performance and complexity. In order to choose an adequate optimization method for a specific application, the particular requirements of this application need to be taken into account. The complexity of pre-stage channel estimation is negligible since the pre-stage channel estimates $\breve{h}$ are obtained in closed form. Furthermore, the pseudoinverse $\left(\boldsymbol{X}_{0}^{H} \boldsymbol{C}_{\boldsymbol{n}}^{-1} \boldsymbol{X}_{0}\right)^{-1} \boldsymbol{X}_{0}^{H} \boldsymbol{C}_{\boldsymbol{n}}^{-1}$ can be computed in advance since the pilot symbols $x_{0}[k]$ and the receive filter $g_{R x}(\tau)$ are known. Hence, the overall complexity is dominated by the optimization algorithm for CPE.

It should be noted here that the only parameter, that is needed for positioning, is $\hat{\theta}_{3}=\hat{\varrho}_{1}=-\hat{\varepsilon}$. All remaining $P-1$ parameters are not relevant for positioning. However, the whole parameter estimate $\hat{\boldsymbol{\theta}}$ is needed to obtain enhanced channel estimates $\hat{\boldsymbol{h}}=\boldsymbol{h}(\hat{\boldsymbol{\theta}})$, which are more reliable than the pre-stage channel estimates $\breve{h}$ due to the usage of a priori information about pulse shaping and receive filtering.

\section{Performance limits-CRLB}

The CRLB corresponds to the best performance that any unbiased estimator can achieve. This means that the covariance matrix of the estimator, $C_{\hat{\theta}}$, is greater than or equal to the inverse of the Fisher information matrix $\boldsymbol{I}(\boldsymbol{\theta})^{-1}[27,28]$ :

$$
\boldsymbol{C}_{\hat{\boldsymbol{\theta}}}-\boldsymbol{I}(\boldsymbol{\theta})^{-1} \geq \mathbf{0},
$$

i.e., the matrix $C_{\hat{\theta}}-\boldsymbol{I}(\boldsymbol{\theta})^{-1}$ is positive semidefinite. If only a single parameter $\theta_{p}, 1 \leq p \leq P$, is considered, the variance of this parameter, which corresponds to the mean squared error (MSE) in case of an unbiased estimator, is greater than or equal to the corresponding diagonal entry of the inverse Fisher information matrix:

$$
\operatorname{MSE}\left(\hat{\theta}_{p}\right)=\left[\boldsymbol{C}_{\hat{\boldsymbol{\theta}}}\right]_{p, p} \geq\left[\boldsymbol{I}(\boldsymbol{\theta})^{-1}\right]_{p, p}=\operatorname{CRLB}\left(\theta_{p}\right) .
$$

According to [28], each entry of the Fisher information matrix is defined as

$$
[\boldsymbol{I}(\boldsymbol{\theta})]_{p, q}=-\mathrm{E}\left\{\frac{\partial^{2} \ln p(\check{\boldsymbol{h}}, \boldsymbol{\theta})}{\partial \theta_{p} \partial \theta_{q}}\right\} .
$$

Employing the Jacobian matrix of the channel function $\boldsymbol{h}(\boldsymbol{\theta})$, which is given by

$$
\boldsymbol{J}(\boldsymbol{\theta})=\frac{\partial \boldsymbol{h}(\boldsymbol{\theta})}{\partial \boldsymbol{\theta}^{T}}=\left[\begin{array}{ccc}
\frac{\partial h_{0}}{\partial \theta_{1}} & \cdots & \frac{\partial h_{0}}{\partial \theta_{P}} \\
\vdots & \ddots & \vdots \\
\frac{\partial h_{L}}{\partial \theta_{1}} & \cdots & \frac{\partial h_{L}}{\partial \theta_{P}}
\end{array}\right],
$$

results in the following Fisher information matrix:

$$
\begin{aligned}
\boldsymbol{I}(\boldsymbol{\theta}) & =2 \operatorname{Re}\left\{\begin{array}{lll}
\boldsymbol{J}(\boldsymbol{\theta})^{H} & \boldsymbol{C}_{\boldsymbol{\eta}}^{-1} & \boldsymbol{J}(\boldsymbol{\theta})
\end{array}\right\} \\
& =2 \operatorname{Re}\left\{\boldsymbol{J}(\boldsymbol{\theta})^{H} \boldsymbol{X}_{0}^{H} \boldsymbol{C}_{\boldsymbol{n}}^{-1} \boldsymbol{X}_{0} \boldsymbol{J}(\boldsymbol{\theta})\right\} .
\end{aligned}
$$

As already mentioned earlier, the estimation of the ToA corresponds to the estimation of the sampling phase and, thus, the only parameter of interest for positioning is $\hat{\theta}_{3}=$ $\hat{\varrho}_{1}=-\hat{\varepsilon}$. Given a certain parameter vector $\boldsymbol{\theta}$, only the corresponding CRLB according to (22) with $p=3$ is considered:

$$
\operatorname{MSE}\left(\hat{\tau}_{1}\right)=\operatorname{MSE}(\hat{\varepsilon})=\left[\boldsymbol{C}_{\hat{\boldsymbol{\theta}}}\right]_{3,3} \geq\left[\boldsymbol{I}(\boldsymbol{\theta})^{-1}\right]_{3,3}=\operatorname{CRLB}(\varepsilon) .
$$

Since there are many possible parameter sets $\theta$, the CRLBs are determined semi-analytically by means of Monte Carlo simulations. In each run of a Monte Carlo simulation, a different channel realization with a different parameter vector $\boldsymbol{\theta}$ is generated and the corresponding Fisher information matrix is determined according to (25). The overall CRLB is given by the expectation of the inverse Fisher information matrices

$$
\operatorname{CRLB}(\varepsilon)=\mathrm{E}\left\{\left[\boldsymbol{I}(\boldsymbol{\theta})^{-1}\right]_{3,3}\right\},
$$

where the expectation is taken with respect to the parameter vector $\boldsymbol{\theta}$. For all channel models examined below, the CRLBs are determined for different oversampling factors over the $\mathrm{SNR}$ in $\mathrm{dB}$. To be more precise, the pilot-to-noise ratio (PNR),

$$
\gamma_{p}=\frac{\mathrm{E}\left\{\left|\sum_{l=0}^{L} h_{l}(\boldsymbol{\theta}) \cdot x_{0}[k-l]\right|^{2}\right\}}{\mathrm{E}\left\{|n[k]|^{2}\right\}},
$$

is taken into account, which is only a fraction of the SNR,

$$
\gamma_{s}=\frac{\mathrm{E}\left\{\left|\sum_{l=0}^{L} h_{l}(\boldsymbol{\theta}) \cdot x[k-l]\right|^{2}\right\}}{\mathrm{E}\left\{|n[k]|^{2}\right\}} .
$$

The relationship between the PNR and the SNR is determined by the pilot layer power: $\gamma_{p}=\rho \cdot \gamma_{s}$. The following simulation setup is applied if not stated otherwise: A burst length of $K^{\prime}=100\left(K=J K^{\prime}\right)$ is assumed and a pseudorandom sequence of BPSK symbols is used as training. A Gaussian pulse shape

$$
p(\tau)=\exp \left(-\left(\tau / T_{s}\right)^{2}\right)
$$

is applied. In order to obtain a causal pulse shape, the Gaussian pulse is shifted by a certain amount $s$. That means that the overall pulse shape is given by

$$
g(\tau)=g_{\mathrm{Tx}}(\tau) * g_{\mathrm{Rx}}(\tau)=p(\tau-s),
$$


which is equally distributed among the pulse shaping and the receive filter. Hence, the autocorrelation of each filter corresponds to a Gaussian pulse: $\psi_{\mathrm{Tx}}(\tau)=\psi_{\mathrm{Rx}}(\tau)=$ $p(\tau)$. In this case, the noise covariance matrix is given by

$$
\left[\boldsymbol{C}_{\boldsymbol{n}}\right]_{i, j}=\sigma_{n}^{2} p((i-j) \mathcal{T})=\sigma_{n}^{2} \exp \left(-\left(\frac{i-j}{J}\right)^{2}\right)
$$

An effective pulse width of $T_{g}=8 T_{s}$ and a shift of $s=$ $0.5 T_{g}=4 T_{s}$ are assumed. In each run of a Monte Carlo simulation, a uniformly distributed random sampling phase $\varepsilon$ is generated in the interval $\left[-0.5 T_{s},+0.5 T_{s}\right]$. The remaining parameters of $\boldsymbol{\theta}$ are generated according to the applied channel model. In all figures below, the quantities concerning timing or delay measures are normalized with respect to the symbol duration, e.g., the CRLB of $\varepsilon$ is normalized to $T_{s}^{2}$. Three different kinds of channel models are considered: A single-path channel, several twopath channels, and different WINNER channels according to [33]. The single-path channel comprises only a lineof-sight (LOS) path and is taken into account since it is the best possible case for positioning and, thus, provides a lower bound for all other channel models. The two-path channels comprise an additional propagation path beside the LOS path. By means of the two-path channel models, the influence of different channel characteristics such as the excess delay, power ratio, and phase offset between the two propagation paths can be investigated. The relationships observed for the two-path channels are the basis for more complex channel models with an arbitrary number of propagation paths. In this case, the mutual relationship between all paths determines the performance. This means that the results obtained for the two-path channels can be used to predict the performance for the WINNER channels, that model wireless radio propagation in urban and indoor environments in a realistic way. Due to the assumption of perfect DIC, the performance limits presented below are not only valid for the system concept proposed by the authors, but can also be applied to other multiplexing techniques like TDM.

\section{Single-path channel}

The single-path channel, which comprises a LOS path only, is taken into account since it is the best possible case for positioning and, thus, provides a lower bound for all other channel models. The channel coefficients of the single-path channel are modeled by

$h_{l}=\exp (\mathrm{j} \Phi) \exp \left(-\left(\frac{l \mathcal{T}+\varepsilon-s}{T_{s}}\right)^{2}\right)=f \exp \left(-\alpha_{l, J}^{2}\right)$ with $f=\exp (\mathrm{j} \Phi), \alpha_{l, J}=l / J+(\varepsilon-s) / T_{s}$. The starting phase $\Phi$ is generated randomly between 0 and $2 \pi$. As there is only a single-path, there are no excess delays and, thus, the channel memory length results in $L^{\prime}=9$ $\left(L=J L^{\prime}\right)$. In Figure 2, the normalized CRLB of $\varepsilon$ for the single-path channel is plotted over the PNR. In Figure 2a, the influence of the burst length $K^{\prime}$ is shown for symbolrate sampling $(J=1)$, whereas in Figure $2 \mathrm{~b}$ the influence of the oversampling factor $J$ is illustrated for a burst length of $K^{\prime}=100$. In all cases, the CRLB decreases with the PNR and is much smaller than one, which corresponds to a small fraction of the squared symbol duration $T_{s}^{2}$. This means that the estimation error is much smaller than the symbol duration $T_{s}$. The larger the burst length, the better the performance: The CRLB improves by approximately $3 \mathrm{~dB}$ if the burst length is doubled (see Figure $2 \mathrm{a}$ ). The same influence of the burst length is observed in all other channel models. Hence, only burst lengths of $K^{\prime}=100$ are considered in the following if not stated otherwise. Furthermore, oversampling provides a slight performance gain as shown in Figure 2b: For all oversampling factors $J \geq 2$, the CRLBs are improved by approximately $0.8 \mathrm{~dB}$ in comparison to symbol-rate sampling. A similar behavior is observed for the remaining channel models as well, i.e., all oversampling factors $J \geq 2$ lead to same performance gain. Hence, only $J=2$ is considered subsequently. The question arises for what reason oversampling provides a performance gain? What is the difference between symbol-rate sampling and oversampling? In order to answer these questions, the CRLB for the single-path channel is examined in more detail. The starting point is the Fisher information matrix given in (25). In order to keep the following investigations manageable, two assumptions are applied which simplify the determination of the Fisher information matrix. First, white noise is assumed for all oversampling factors and, second, the burst length is assumed to be large. In this case, the inverse covariance matrix of the channel estimation error can be approximated by a scaled identity matrix

$$
\boldsymbol{C}_{\eta}^{-1}=\boldsymbol{X}_{0}^{H} \boldsymbol{C}_{\boldsymbol{n}}^{-1} \boldsymbol{X}_{0} \approx \gamma \cdot \mathcal{I}
$$

with scaling factor $\gamma=\left(K^{\prime}-L^{\prime}\right) /\left(J \sigma_{n}^{2}\right)$. This leads to an approximate Fisher information matrix

$$
\boldsymbol{I}(\boldsymbol{\theta}) \approx 2 \gamma \cdot \operatorname{Re}\left\{\boldsymbol{J}(\boldsymbol{\theta})^{H} \boldsymbol{J}(\boldsymbol{\theta})\right\} .
$$

Hence, only the Jacobian matrix needs to be determined. The partial derivatives of the channel coefficients are given by

$$
\begin{aligned}
& \frac{\partial h_{l}}{\partial \theta_{1}}=\exp \left(-\alpha_{l, J}^{2}\right), \quad \frac{\partial h_{l}}{\partial \theta_{2}}=\mathrm{j} \exp \left(-\alpha_{l, J}^{2}\right), \\
& \frac{\partial h_{l}}{\partial \theta_{3}}=2 f \cdot \exp \left(-\alpha_{l, J}^{2}\right) \cdot \alpha_{l, J} \cdot
\end{aligned}
$$


(a)

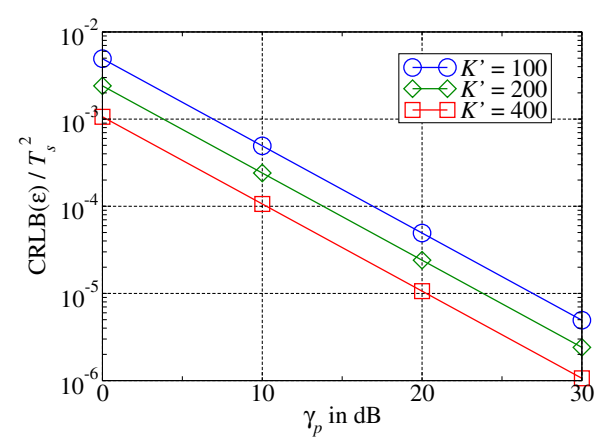

(b)

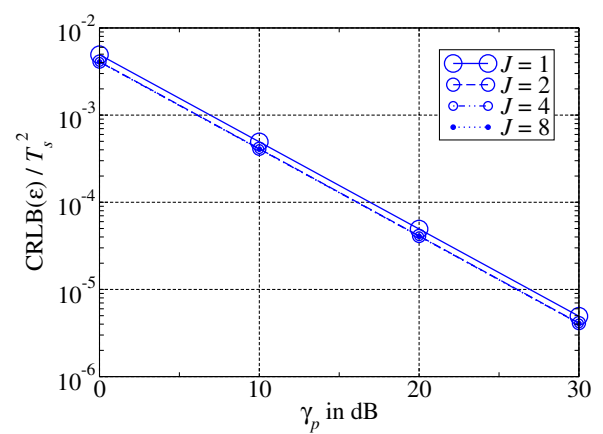

Figure 2 Normalized CRLB of $\varepsilon$ versus PNR for the single-path channel. (a) Influence of the burst length $K^{\prime}(J=1)$. (b) Influence of the oversampling factor $J\left(K^{\prime}=100\right)$.

Multiplying the Hermitian conjugate of the Jacobian matrix with the Jacobian matrix itself and taking the real part of the result leads to a Fisher information matrix of the following form:

$$
\boldsymbol{I}(\boldsymbol{\theta}) \approx 2 \gamma \cdot\left[\begin{array}{ccc}
A & 0 & B \\
0 & A & C \\
B & C & D
\end{array}\right]
$$

with

$$
\begin{aligned}
& A=\sum_{l=0}^{L}\left\{\exp \left(-\alpha_{l, J}^{2}\right)\right\}^{2}, \\
& B=2 \operatorname{Re}\{f\} \sum_{l=0}^{L}\left\{\exp \left(-\alpha_{l, J}^{2}\right)\right\}^{2} \cdot \alpha_{l, J}, \\
& C=2 \operatorname{Im}\{f\} \sum_{l=0}^{L}\left\{\exp \left(-\alpha_{l, J}^{2}\right)\right\}^{2} \cdot \alpha_{l, J}, \\
& D=4|f|^{2} \sum_{l=0}^{L}\left\{\exp \left(-\alpha_{l, J}^{2}\right)\right\}^{2} \cdot \alpha_{l, J}^{2} .
\end{aligned}
$$

Inverting the matrix in (37) results in
The CRLB of the sampling phase corresponds to the third main diagonal entry of the above matrix

$\operatorname{CRLB}(\varepsilon)$

$$
\begin{aligned}
& =\left[\boldsymbol{I}(\boldsymbol{\theta})^{-1}\right]_{3,3} \approx \frac{1}{2 \gamma} \cdot \frac{A}{A D-B^{2}-C^{2}} \\
& =\frac{1}{8 \gamma|f|^{2}} . \\
& \times \frac{\sum_{l=0}^{L}\left\{\exp \left(-\alpha_{l, J}^{2}\right)\right\}^{2}}{\left[\sum_{l=0}^{L}\left\{\exp \left(-\alpha_{l, J}^{2}\right)\right\}^{2}\right]\left[\sum_{l=0}^{L}\left\{\exp \left(-\alpha_{l, J}^{2}\right)\right\}^{2} \cdot \alpha_{l, J}^{2}\right]-\left[\sum_{l=0}^{L}\left\{\exp \left(-\alpha_{l, J}^{2}\right)\right\}^{2} \cdot \alpha_{l, J}\right]_{(43)}^{2}} .
\end{aligned}
$$

Since $|f|^{2}$ is always one in the single-path channel, the difference between symbol-rate sampling and oversampling must depend somehow on the sums over the exponential terms and, thus, on the sampling phase itself. In Figure 3, the influence of the sampling phase on the approximate CRLB is illustrated: In Figure 3a, the approximate CRLB is plotted over the sampling phase $\varepsilon$ for symbol-rate sampling and oversampling with $J=2$ for $\gamma_{p}=10 \mathrm{~dB}$. For oversampling with $J=2$, the approximate CRLB is constant and does not depend on $\varepsilon$. In contrast, the approximate CRLB varies with the sampling phase for symbol-rate sampling. Thus, there is a considerable difference between symbol-rate sampling and oversampling. The same behavior can be observed for the average channel power divided by the oversampling factor,

$$
\boldsymbol{I}(\boldsymbol{\theta})^{-1} \approx \frac{1}{2 \gamma} \cdot \frac{1}{A\left(A D-B^{2}-C^{2}\right)}\left[\begin{array}{ccc}
A D-C^{2} & B C & -A B \\
B C & A D-B^{2} & -A C \\
-A B & -A C & A^{2}
\end{array}\right] .
$$

$$
\frac{1}{J} \mathrm{E}\left\{\sum_{l=0}^{L}\left|h_{l}(\boldsymbol{\theta})\right|^{2}\right\}=\frac{1}{J} \mathrm{E}\left\{\sum_{l=0}^{L}\left\{\exp \left(\alpha_{l, J}^{2}\right)\right\}^{2}\right\}
$$


(a)

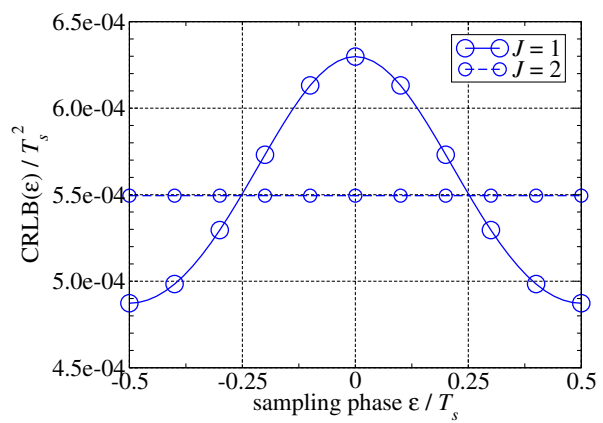

(b)

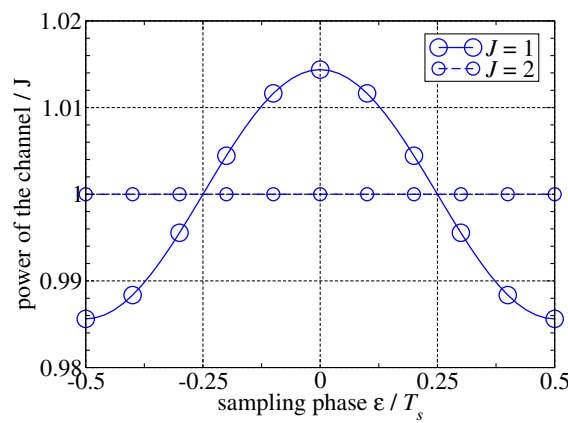

Figure 3 Influence of the sampling phase on the approximate normalized CRLB. (a) Approximate normalized CRLB of $\varepsilon$ versus the sampling phase with $\gamma_{p}=10 \mathrm{~dB}$. (b) Average channel power divided by the oversampling factor vs. the sampling phase.

which corresponds to the numerator in (43). The average channel power according to (44) is plotted in Figure 3b: For oversampling with $J=2$, the power of the channel is constant and, thus, independent of the sampling phase. For symbol-rate sampling, in contrast, the power of the channel is a function of the sampling phase: The highest power is obtained for $\varepsilon=0$ (perfect synchronization), while the smallest power occurs for $|\varepsilon|=0.5 T_{s}$. This means that non-perfect synchronization leads to a power loss concerning symbol-rate sampling. Similar results have already been reported by the authors in [6] for a rectangular and a root raised cosine pulse shape.

Now, it is clear that there is a considerable difference between symbol-rate sampling and oversampling. But the question remains: How does this difference lead to the observed performance gain of oversampling? In order to answer this question, it is necessary to return to the exact Fisher information matrix described by (25) and the corresponding exact CRLB. How does the behavior of the CRLB change if the exact instead of the approximate version is taken into account? In Figure 4a, both types of the
CRLB are plotted over the sampling phase $\varepsilon$ for $K^{\prime}=$ $100, \gamma_{p}=10 \mathrm{~dB}$ and $J=1,2$. The curves are labeled with "A" for approximate and "E" for exact. In order to have a closer look at the different behavior of the approximate and the exact CRLB, the normalized difference between the symbol-rate sampled and the oversampled CRLB according to

$$
\frac{\left.\operatorname{CRLB}(\varepsilon)\right|_{J=1}-\left.\operatorname{CRLB}(\varepsilon)\right|_{J=2}}{\left.\operatorname{CRLB}(\varepsilon)\right|_{J=1}},
$$

is plotted for each type in Figure $4 \mathrm{~b}$. For the approximate and the exact CRLB, the shape of the corresponding curves is basically the same. The difference between the approximate CRLB and the exact CRLB lies in the fact that the curves of the exact CRLB are shifted downward with the shift for symbol-rate sampling being smaller than the shift for oversampling. This means that the curves of the normalized difference between the CRLBs are shifted upward. A slight deviation is observed in case of the (a)

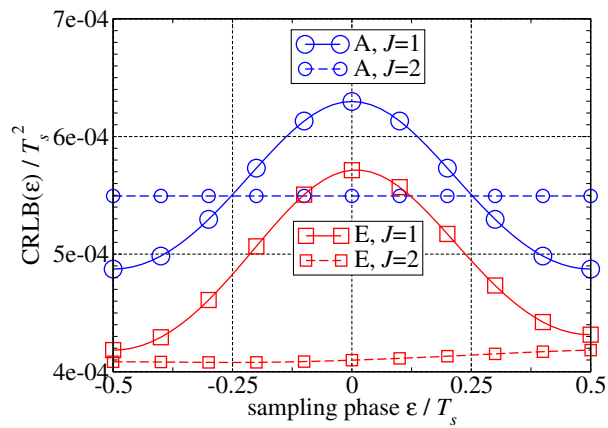

(b)

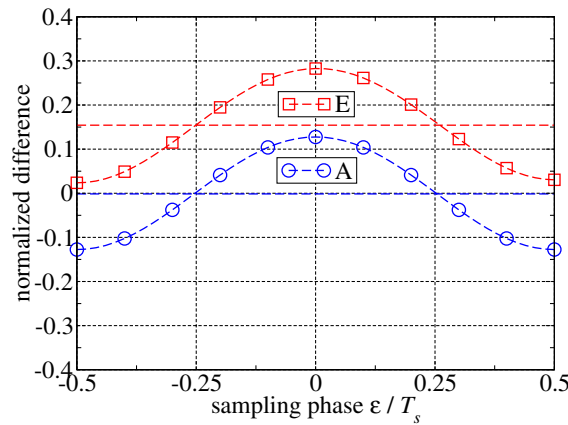

Figure 4 Comparison of approximate and exact normalized CRLBs vs. the sampling phase with $\gamma_{p}=10 \mathrm{~dB}$. The curves are labeled with " $\mathrm{A}$ " for approximate and " $E$ " for exact. (a) Normalized CRLB of $\varepsilon$ with $K^{\prime}=100$. (b) Normalized difference of CRLBs with $K^{\prime}=100$. 
exact CRLB for $J=2$ : In contrast to the approximate CRLB for oversampling, the exact CRLB for oversampling is not absolutely constant over the whole range of sampling phases, but it slightly increases for $\varepsilon \geq 0$ (see Figure 4a). This effect is due to the short burst length of $K^{\prime}=100$. For larger burst lengths (e.g., $K^{\prime}=400$ ), this effect is not present anymore and the exact CRLB for $J=2$ is absolutely constant over the whole sampling phase range as well. The most interesting aspect is revealed by the mean of the normalized difference, which is shown as a straight line in Figure $4 \mathrm{~b}$. If white noise is assumed for all oversampling factors ("A"), then the mean of the normalized difference (45) equals zero in all cases. That means that on average there is no difference between symbol-rate sampling and oversampling such that the CRLBs determined via Monte Carlo simulations (with a random sampling phase) are the same. In case of colored noise ("E"), the mean of the normalized difference (45) is approximately 0.16 for $K^{\prime}=100$, i.e., the exact CRLBs for symbol-rate sampling and oversampling differ on average by a small amount. This difference leads to the oversampling gain which has been observed in Figure 2b.

Last but not least, it is interesting to note that the mean of the normalized difference as well as the oversampling gain vary with the burst length $K^{\prime}$. In Figure 5 , the influence of the burst length $K^{\prime}$ is examined in more detail: In Figure 5a, the CRLB of $\varepsilon$ is plotted for symbol-rate sampling and oversampling with different burst lengths of $K^{\prime}=100$ and $K^{\prime}=400$. As already mentioned before, the performance gain of oversampling is approximately $0.8 \mathrm{~dB}$ for $K^{\prime}=100$. For $K^{\prime}=400$, this gain is increased to approximately $1 \mathrm{~dB}$. For comparison, the mean of the normalized difference is plotted over the burst length $K^{\prime}$ in Figure 5b. The circles denote the simulated values, while the dashed line corresponds to a fitted curve. It is observed that the mean normalized difference increases with the burst length and saturates around
0.223 , i.e., from approximately $K^{\prime}=800$ upward the mean normalized difference is constant. The mean normalized difference for $K^{\prime}=400$ is approximately 0.2 . Interestingly, the ratio of the performance gains corresponds to the ratio of the mean normalized differences: $0.8 / 1 \mathrm{~dB}=0.8=0.16 / 0.2$. Thus, the mean of the normalized difference translates into the performance gain with a positive factor. As the mean normalized difference saturates around 0.223 , the maximum achievable oversampling gain is approximately $1.115 \mathrm{~dB}$ in a single-path channel.

\section{Two-path channels}

Two-path channels represent the simplest form of a multipath channel. Since block fading is assumed, a two-path channel can be fully characterized according to

$$
\begin{aligned}
h_{l}= & a_{1} \exp \left(\mathrm{j} \Phi_{1}\right) \exp \left(-\left(\frac{l \mathcal{T}+\varepsilon-s}{T_{s}}\right)^{2}\right)+a_{2} \exp \left(\mathrm{j} \Phi_{2}\right) \\
& \times \exp \left(-\left(\frac{l \mathcal{T}+\varepsilon-s-v_{2}}{T_{s}}\right)^{2}\right) .
\end{aligned}
$$

By means of the two-path channel models, the influence of different channel characteristics such as the excess delay $\nu_{2}$, power ratio $\mathcal{P}=a_{1}^{2} / a_{2}^{2}$, and phase offset $\Delta \Phi=$ $\Phi_{2}-\Phi_{1}$ between the two propagation paths can be investigated. The maximum possible excess delay is fixed to $v_{2}^{\max }=2 T_{s}$, which leads to a channel memory length of $L^{\prime}=11\left(L=J L^{\prime}\right)$. At first, the CRLB of $\varepsilon$ is examined over PNR, where the power ratio and the phase offset are generated randomly in the intervals $[0.1,10]$ and $[0,2 \pi]$, respectively. Concerning the excess delay, two different scenarios are considered: one with small excess delays $\left(v_{2} / T_{s} \in[0.05,1]\right)$ and one with large excess delays $\left(v_{2} / T_{s} \in[1,2]\right)$. The corresponding CRLBs for different (a)

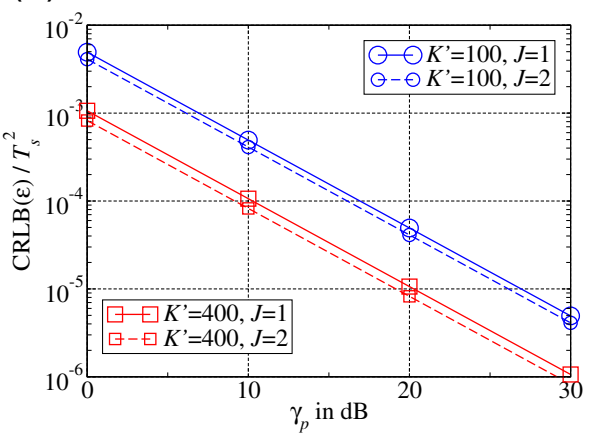

(b)

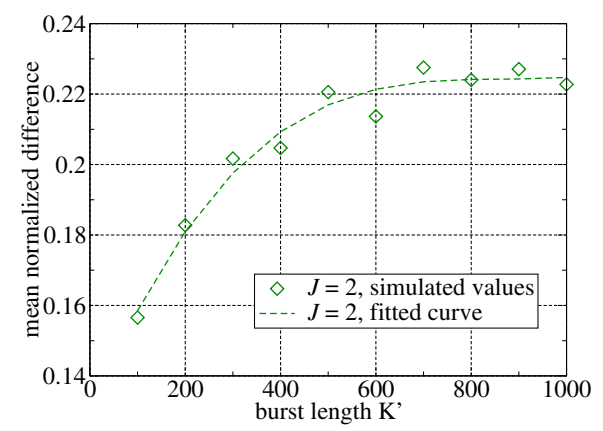

Figure 5 Influence of the burst length $K^{\prime}$ on the oversampling gain. (a) Normalized CRLB of $\varepsilon$ Vs. PNR for different oversampling factors and burst lengths. (b) Mean normalized difference of CRLBs vs. burst length $K^{\prime}$. 
oversampling factors are shown in Figure 6. The overall performance decreases with respect to the LOS channel (as expected) but the oversampling gain increases. Both, the overall performance and the oversampling gain, depend on the channel model: The smaller the excess delay, the worse is the CRLB and the larger is the oversampling gain. For the channel with small excess delay, the gain is approximately $6.5 \mathrm{~dB}$, whereas it is only $2.6 \mathrm{~dB}$ for the channel with large excess delay. In order to have a closer look at this dependence, the CRLBs are determined over the excess delay for a fixed PNR $\left(\gamma_{p}=10 \mathrm{~dB}\right)$. Similarly, the influence of the phase offset $\Delta \Phi$ and the power ratio $\mathcal{P}$ is investigated.

In contrast to the LOS channel, the approximate Fisher information matrix according to (35) and the corresponding approximate CRLB are not considered here. Due to the increased size of the Fisher information matrix $(6 \times 6$ instead of $3 \times 3)$, the resulting formula for the approximate CRLB consists of many different terms and is, therefore, less concise than in the case of the LOS channel. Two basic simulation setups are applied, whose characteristics are tabulated in Table 1. The parameter of interest is varied, while the other parameters are fixed to the values given in Table 1. The corresponding CRLBs and their normalized differences according to (45) are shown on the left-hand side and on the right-hand side of Figure 7, respectively. The curves are labeled with "S1" for the first setup and "S2" for the second setup. The mean normalized difference of the LOS channel (for $K^{\prime}=100$ ) is given for comparison in Figure $7 \mathrm{~b}, \mathrm{~d}, \mathrm{f}$ since it corresponds to a lower bound concerning the normalized difference of the two-path channels. In the first row of Figure 7, the influence of the excess delay $v_{2}$ is illustrated for both setups, while the influence of the phase offset $\Delta \Phi$ and the power ratio $\mathcal{P}$ are shown in the second and third row, respectively. The overall performance and the oversampling gain

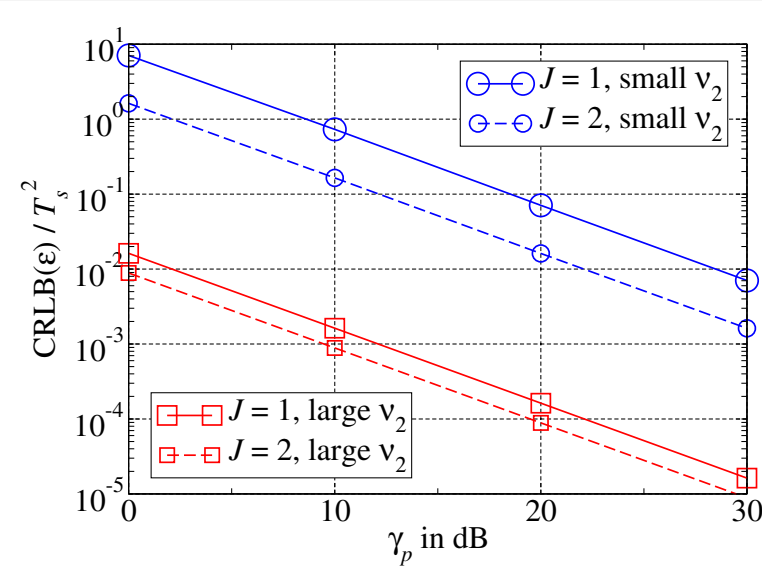

Figure 6 Normalized CRLB of $\varepsilon$ versus PNR for the two-path channels with small and large excess delay $v_{2}$.
Table 1 Basic simulation setups for the two-path channel models

\begin{tabular}{lll}
\hline Parameter & Setup 1 & Setup 2 \\
\hline$\nu_{2} / T_{s}$ & 0.5 & 1.5 \\
$\mathcal{P}$ & 1.0 & 5.0 \\
$\Delta \Phi$ & $0^{\circ}$ & $90^{\circ}$ \\
\hline
\end{tabular}

strongly depend on the simulation setup and the channel characteristic of interest. It is obvious that the first setup corresponds to a kind of worst case concerning parameter estimation, whereas the second setup represents a kind of best case scenario. For the first setup, oversampling can provide a significant gain over symbol-rate sampling. This gain is not influenced by the power ratio (see Figure $7 \mathrm{f}$ ), while it mainly depends on the excess delay $v_{2}$ (see Figure 7b): The smaller the excess delay, the larger is the oversampling gain. Hence, oversampling proves especially helpful in dense multipath scenarios. Furthermore, the phase offset $\Delta \Phi$ has a significant impact on the oversampling gain, which is highest when both propagations paths have the same or the opposite phase (see Figure 7d). As mentioned above, the normalized difference translates into the oversampling gain with a positive factor. Thus, the larger the normalized difference, the larger is the oversampling gain.

Figure 7 does not only illustrate the dependence of the oversampling gain on the channel characteristics, but gives also an insight into the relation between these characteristics and the overall performance. With decreasing excess delay $v_{2}$, the CRLB increases as it becomes more difficult to separate the two propagation paths. Similarly, the CRLB is worst if the paths are in phase $\left(\Delta \Phi=0^{\circ}\right)$ or have an opposite phase $\left(\Delta \Phi=180^{\circ}\right)$. The smaller the power ratio $\mathcal{P}$, the smaller is the amplitude of the first path in comparison to the second path and the more likely it is that the delay of the first path, namely the sampling phase, is estimated wrongly. Thus, the influence of the channel characteristics on the overall performance can be summarized as follows: Dense multipath scenarios with similar or opposite phases and a small power ratio are most challenging. The more challenging it is to estimate the sampling phase, the higher is the gain due to oversampling. Hence, the application of oversampling (with $J=2$ ) is highly recommended.

\section{WINNER channels}

The WINNER channels described in [33] represent realistic scenarios with a high number of propagation paths. Many different propagation scenarios are considered including rural, suburban and urban as well as indoor scenarios. Generally, the WINNER channel models are suited for the evaluation of multiple-input multiple-output systems. However, only the case of single-input single-output 
(a)

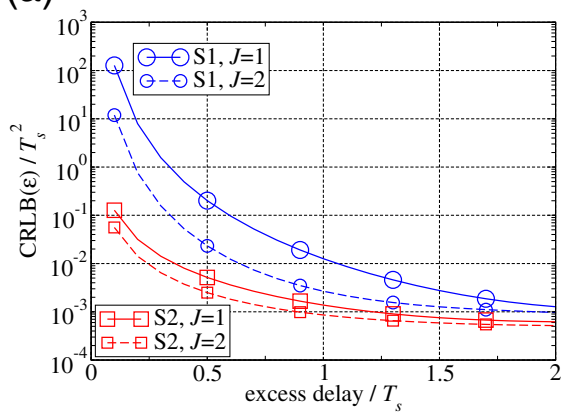

(c)

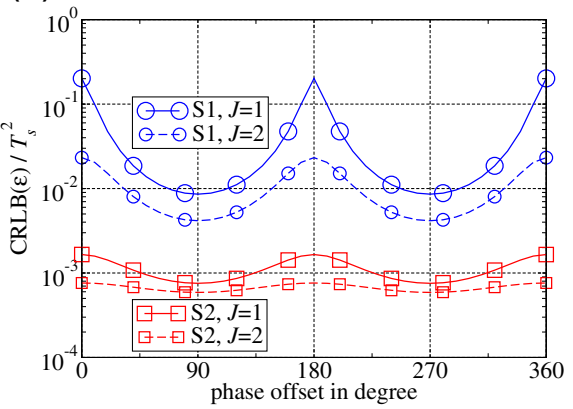

(e)

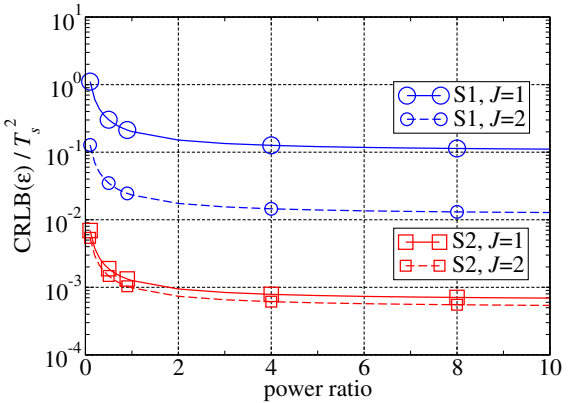

(b)

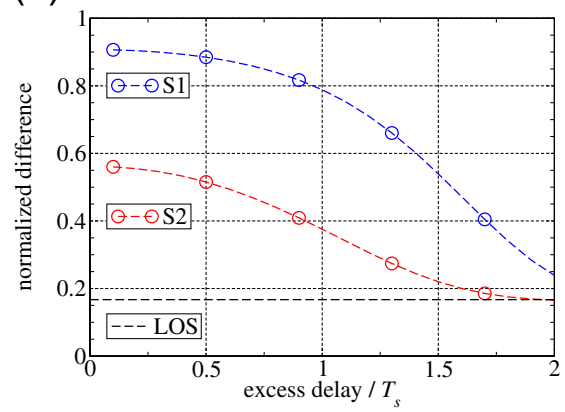

(d)

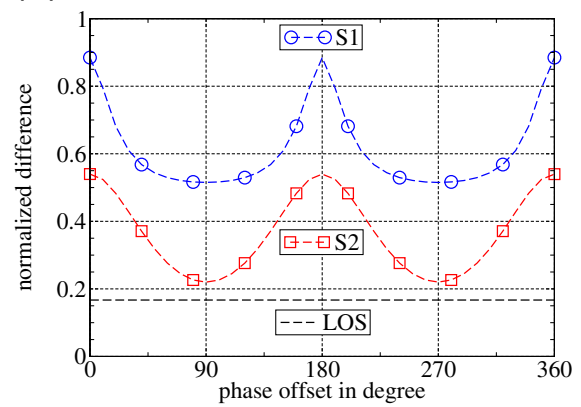

(f)

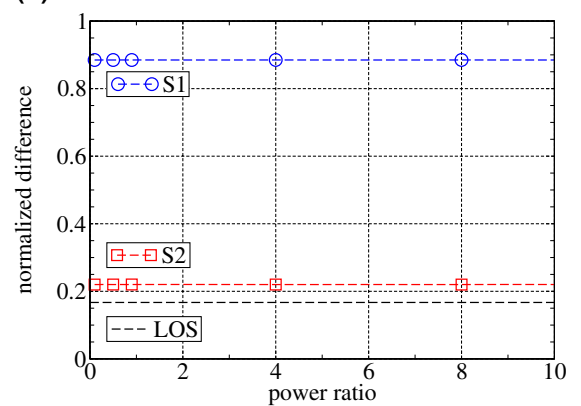

Figure 7 Comparison of normalized CRLBs and the normalized difference between CRLBs for different channel characteristics with $\gamma_{p}=10$ dB. The curves are labeled with "S1" for the first setup and "S2" for the second setup. (a) Normalized CRLB of $\varepsilon$ versus excess delay. (b) Normalized difference of CRLBs versus excess delay. (c) Normalized CRLB of $\varepsilon$ versus phase offset. (d) Normalized difference of CRLBs versus phase offset. (e) Normalized CRLB of $\varepsilon$ versus power ratio. (f) Normalized difference of CRLBs versus power ratio.

(SISO) is considered here. Two types of channel models are presented in [33]: The generic model, that is suited for system level simulations, and the clustered delay line $(C D L)$ model, that is reduced in complexity for fast link level simulations. In this contribution, the CDL models are utilized. The parameters of the CDL models like the excess delay or the average power of a multipath component are fixed and tabulated. A single multipath component is called cluster in [33]. With the Gaussian pulse shape in (30) and the block fading assumption, the channel coefficients for WINNER channel models with $I$ clusters are given as

$$
h_{l}=\sum_{i=1}^{I} f_{i} \cdot \exp \left(-\left(\frac{l \mathcal{T}+\varepsilon-s-v_{i}}{T_{s}}\right)^{2}\right),
$$

where the complex amplitudes $f_{i}$ are determined by the superposition of $R=20$ rays according to

$$
f_{i}=A_{i} \cdot \frac{1}{\sqrt{R}} \sum_{r=1}^{R} \exp \left(\mathrm{j} \Phi_{i, r}\right) .
$$

If a LOS component is present, the first cluster consists of $R=21$ rays. Each cluster is assigned a normalized amplitude $A_{i}$ that is computed from the tabulated cluster powers $P_{i}$ as

$$
A_{i}=\sqrt{P_{i} / \sum_{i=1}^{I} P_{i}}
$$

in order to achieve a power normalization. The starting phases $\Phi_{i, r}$ are determined randomly between 0 and $2 \pi$ 
for each ray in every cluster. Three different WINNER channel models are considered in this paper: typical urban micro-cell (B1-LOS), large indoor hall (B3-LOS) and typical urban macro-cell (C2-LOS). For an exact description of these scenarios and the corresponding parameter tables please refer to [33]. ${ }^{\mathrm{a}}$ The most important parameters of the WINNER channel models are summarized in the upper part of Table 2. In addition to the number of clusters $I$ and the number of parameters $P$, the Rician factor $K_{R}$ as well as the excess delays $v_{2}$ and $v_{I}$ are listed. Furthermore, the minimum delay difference, $\min \left\{v_{i}-v_{i-1}\right\}$, and the average delay difference, $\mathrm{E}\left\{v_{i}-v_{i-1}\right\}$, between neighboring paths is determined. For the minimum delay difference, the cluster number for which this difference occurs is given. Until now, only normalized excess delays $v_{2} / T_{s}$ have been considered. Thus, the preceding results are valid for any choice of the symbol duration $T_{s}$. In case of the WINNER channel models, the excess delays are given as absolute values in ns. Hence, the simulation results depend on the choice of the symbol duration $T_{s}$. Here, $T_{s}=10 \mathrm{~ns}$ is considered. In the lower part of Table 2, the channel memory length $L^{\prime}$ for symbol-rate sampling $\left(L=J L^{\prime}\right)$ as well as the relative excess delays and relative delay differences for $T_{s}=10 \mathrm{~ns}$ are tabulated.

In the WINNER channel models, parameter estimation is much more challenging in comparison to the two-path channel models since a much larger number of propagation paths $I>2$ is considered. Thus, the dimension of the estimation process $P=3 I$ increases significantly. However, the relationships observed for the two-path channels are the basis for more complex channel models with an arbitrary number of propagation paths like the WINNER channel models. In this case, the mutual relationship between all paths determines the performance, where the relationship between the first and the second path is of special importance. A performance prognosis can be given based on the results obtained for the two-path channel models: Dense multipath scenarios with similar or opposite phases and a small power ratio are most challenging. Due to the superposition of several rays per cluster with random starting phases, the phase offsets and power ratios of all paths are random and can not be influenced. Nevertheless, the Rician factor is a valuable indicator since it is defined as the average power ratio of the LOS path and the scattered components. The smaller the Rician factor, the smaller is the average LOS power and the worse the performance should be. From this point of view, the B3-LOS channel is most challenging. Furthermore, the relative excess delays and delay differences are of interest. Especially, the relative excess delay of the first multipath component $v_{2} / T_{s}$ is important since the corresponding pulse overlaps the most with the LOS component. For $T_{s}=10 \mathrm{~ns}$, the relative excess delays of the first multipath component are in a reasonable range. The same is valid for the relative minimum delay differences. Again, the B3-LOS is most challenging with respect to delay differences because a very small delay difference of $3.5 \mathrm{~ns}$ occurs twice. Taking all parameters into account, the worst performance is expected for the B3-LOS channel (large indoor hall), whereas the best performance is predicted for the B1-LOS channel (urban micro-cell). The CRLBs of all three WINNER channels for symbol-rate sampling and oversampling with $J=2$ are shown in Figure 8 . The above performance prediction is met by all simulation results: The best and worst performance is obtained for the B1-LOS and the B3-LOS channel, respectively. For oversampling with $J=2$ (dashed lines), the CRLBs are even better than the corresponding CRLB for the two-path channel with small excess delay. The performance of the B1-LOS channel is even similar to the

Table 2 Selected parameters of the WINNER channels

\begin{tabular}{|c|c|c|c|c|}
\hline & Parameter & $\begin{array}{l}\text { B1-LOS } \\
\text { (urban micro-cell) }\end{array}$ & $\begin{array}{l}\text { B3-LOS } \\
\text { (large indoor hall) }\end{array}$ & $\begin{array}{l}\text { C2-LOS } \\
\text { (urban macro-cell) }\end{array}$ \\
\hline & 1 & 8 & 10 & 8 \\
\hline & P & 24 & 30 & 24 \\
\hline & $K_{R}(\mathrm{~dB})$ & 3.3 & 2.0 & 7.0 \\
\hline & $v_{2}$ (ns) & 33.5 & 3.5 & 3.5 \\
\hline & $v_{l}(\mathrm{~ns})$ & 460.0 & 280.0 & 220.0 \\
\hline & $\min \left\{v_{i}-v_{i-1}\right\}$ (ns) & $8.5(i=4)$ & $3.5(i=2 ; 6)$ & $1.5(i=6)$ \\
\hline & $\mathrm{E}\left\{v_{i}-v_{i-1}\right\}$ (ns) & 65.7 & 31.1 & 32.85 \\
\hline \multirow{5}{*}{$T_{s}=10 \mathrm{~ns}$} & $L^{\prime}$ & 55 & 37 & 31 \\
\hline & $v_{2} / T_{s}$ & 3.35 & 0.35 & 0.35 \\
\hline & $v_{l} / T_{s}$ & 46.00 & 28.00 & 22.00 \\
\hline & $\min \left\{v_{i}-v_{i-1}\right\} / T_{s}$ & 0.85 & 0.35 & 0.15 \\
\hline & $\mathrm{E}\left\{v_{i}-v_{i-1}\right\} / T_{s}$ & 6.57 & 3.11 & 3.285 \\
\hline
\end{tabular}




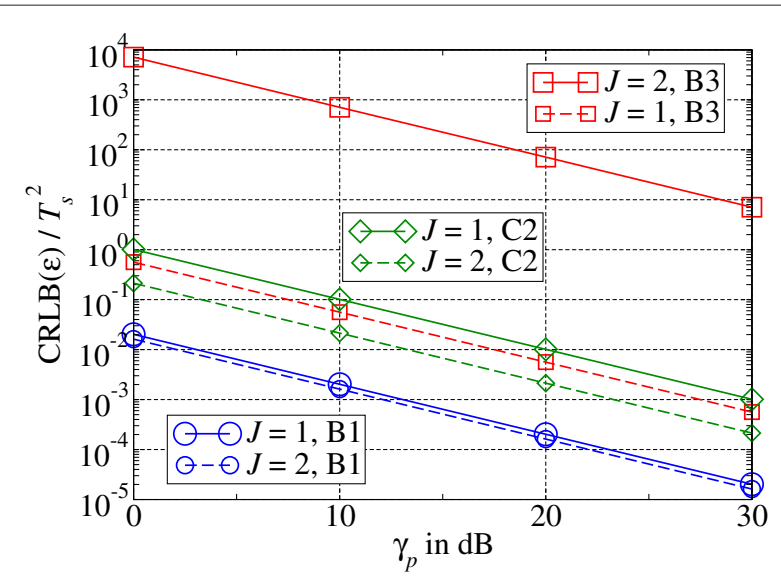

Figure 8 Normalized CRLB of $\varepsilon$ versus PNR for the WINNER channels B1, B3 and C2 with $T_{s}=10 \mathrm{~ns}$.

performance of the two-path channel with large excess delay. In contrast, a significant performance degradation is observed for symbol-rate sampling. For the B3-LOS channel, the normalized CRLB at $\gamma_{p}=0 \mathrm{~dB}$ is very high with a value close to $10^{4}$. This means that oversampling gains larger than $30 \mathrm{~dB}$ are possible in case of the WINNER channel models. Hence, it is highly recommended to apply oversampling with $J=2$ because accuracies well below the symbol duration can only be obtained for oversampling.

The symbol duration of $T_{s}=10 \mathrm{~ns}$ has been chosen for the simulations in order to achieve reasonable values for the relative excess delays. With increasing symbol duration $T_{s}$, the relative excess delays and delay differences decrease. Very small delay differences lead to illconditioned (or even rank-deficient) Fisher information matrices, that are difficult to invert. In order to avoid a complete failure of the matrix inversion, singular value decomposition as described in [32, p. 62ff.] can be applied. However, the corresponding CRLBs degrade significantly if the relative delay differences become too small. In this case, the channel models are not adequate anymore and should be revised because neighboring clusters are not resolvable by any means and act like additional rays that contribute to a single cluster. This means, that clusters with nearly the same relative excess delay should be combined to a single cluster. In this way, the number of clusters is reduced, the new clusters are resolvable and meaningful CRLBs can be determined again.

\section{Impact of the performance limits for CPE on the overall positioning process}

As already mentioned in the introduction part of this article, positioning is typically performed in two steps, namely parameter estimation and position estimation. Until now, only the first step (CPE) has been examined. In the following, the impact of the performance limits for CPE on the second step is discussed. Hence, several links between different reference objects (ROs) and a mobile station (MS) in a certain geometrical setup have to be taken into account. For a better understanding, the positioning problem for localization based on the ToA is shortly introduced and a corresponding CRLB is derived. Two-dimensional positioning is considered for that purpose, i.e., at least three ROs are needed to determine the position of the MS. An extension to three-dimensional localization is straight forward.

The MS's position, that shall be estimated from the ToAs, is denoted by $\boldsymbol{p}=\left[p_{1}, p_{2}\right]^{T}=[x, y]^{T}$, while the known locations of the ROs are denoted by $\boldsymbol{p}_{b}=\left[p_{b, 1}, p_{b, 2}\right]^{T}=\left[x_{b}, y_{b}\right]^{T}, 1 \leq b \leq B$, where $B$ is the number of reference objects. The true distance between the MS and the $b$ th RO is a nonlinear function of the current MS's position $\boldsymbol{p}$ and can be determined according to

$$
d_{b}(\boldsymbol{p})=\sqrt{\left(x-x_{b}\right)^{2}+\left(y-y_{b}\right)^{2}} .
$$

These distances are estimated via the ToAs $\hat{\tau}_{b, 1}$ based on $\hat{d}_{b}=\hat{\tau}_{b, 1} \cdot \mathrm{c}$, where $\mathrm{c}$ is the speed of light. The estimated distances $\hat{\boldsymbol{d}}=\left[\hat{d}_{1}, \ldots, \hat{d}_{B}\right]^{T}$ are called pseudoranges since they consist of the true distances $\boldsymbol{d}(\boldsymbol{p})=\left[d_{1}(\boldsymbol{p}), \ldots, d_{B}(\boldsymbol{p})\right]^{T}$ and estimation errors $\boldsymbol{e}=\left[e_{1}, \ldots, e_{B}\right]^{T}$ with covariance matrix $\boldsymbol{C}_{\boldsymbol{e}}=$ diag $\left(\sigma_{e_{1}}^{2}, \ldots, \sigma_{e_{B}}^{2}\right)$ :

$$
\hat{d}=d(p)+e .
$$

Again, an ML estimator can be applied to estimate the position of the MS:

$$
\begin{aligned}
\hat{\boldsymbol{p}} & =\arg \min _{\tilde{\boldsymbol{p}}}\left\{(\hat{\boldsymbol{d}}-\boldsymbol{d}(\tilde{\boldsymbol{p}}))^{T} \boldsymbol{C}_{\boldsymbol{e}}^{-1}(\hat{\boldsymbol{d}}-\boldsymbol{d}(\tilde{\boldsymbol{p}}))\right\} \\
& =\arg \min _{\tilde{\boldsymbol{p}}}\left\{\Omega_{\hat{d}}(\tilde{\boldsymbol{p}})\right\}
\end{aligned}
$$

Similar to the ML estimator for CPE, the metric $\Omega_{\hat{\boldsymbol{d}}}(\tilde{\boldsymbol{p}})$ for position estimation is nonlinear due to the nonlinear distance function (50). Hence, an optimization algorithm has to be applied similar to the case of CPE. Often, a Gauss-Newton approach known as Taylor series algorithm $[34,35]$ is utilized for positioning. But for positioning, there are additionally some approximative, non-iterative estimators like the weighted least-squares algorithm available [36,37].

As in the case of CPE, a CRLB can be determined for positioning, that corresponds to the best performance that any unbiased estimator can achieve. This means that the covariance matrix of the position estimator $C_{\hat{p}}$ is 
greater than or equal to the inverse of the Fisher information matrix $\boldsymbol{I}^{-1}(\boldsymbol{p})[27,28]$ :

$$
C_{\hat{\boldsymbol{p}}}-\boldsymbol{I}^{-1}(\boldsymbol{p}) \geq \mathbf{0}
$$

i.e., the matrix $C_{\hat{p}}-I^{-1}(p)$ is positive semi-definite. The CRLB for positioning is given by the trace of the inverse Fisher information matrix

$$
\operatorname{CRLB}(\boldsymbol{p})=\operatorname{tr}\left\{\boldsymbol{I}^{-1}(\boldsymbol{p})\right\}=\sum_{i=1}^{2}\left[\boldsymbol{I}^{-1}(\boldsymbol{p})\right]_{i, i} .
$$

Similar to the derivations for CPE, the following expression is obtained for the Fisher information matrix in case of positioning:

$$
\boldsymbol{I}(\boldsymbol{p})=2 \cdot \boldsymbol{J}^{T}(\boldsymbol{p}) \boldsymbol{C}_{\boldsymbol{e}}^{-1} \boldsymbol{J}(\boldsymbol{p}),
$$

where $\boldsymbol{J}(\boldsymbol{p})$ denotes the Jacobian matrix of the distance function $\boldsymbol{d}(\boldsymbol{p})$ given by

$$
\boldsymbol{J}(\boldsymbol{p})=\frac{\partial \boldsymbol{d}(\boldsymbol{p})}{\partial \boldsymbol{p}^{T}}=\left[\frac{\partial \boldsymbol{d}(\boldsymbol{p})}{\partial x}, \frac{\partial \boldsymbol{d}(\boldsymbol{p})}{\partial y}\right]=\left[\begin{array}{ccc}
\frac{\partial d_{1}(\boldsymbol{p})}{\partial x} & \cdots & \frac{\partial d_{1}(\boldsymbol{p})}{\partial y} \\
\vdots & \ddots & \vdots \\
\frac{\partial d_{B}(\boldsymbol{p})}{\partial x} & \cdots & \frac{\partial d_{B}(\boldsymbol{p})}{\partial y}
\end{array}\right],
$$

Since the positions of all involved ROs are required in order to determine the Fisher information matrix, the positioning accuracy depends on the geometry between the ROs and the MS. In order to separate the influence of the geometry from the influence of the estimation errors $\boldsymbol{e}$ on the positioning accuracy, the GDOP is taken into account [10]. The GDOP is defined as the square root of the CRLB, given the assumption that all pseudoranges are affected by the same error variance $\sigma_{e_{b}}^{2}=1,1 \leq b \leq B$ (i.e. $\left.C_{e}=\mathcal{I}\right)$ :

$$
\operatorname{GDOP}(\boldsymbol{p})=\sqrt{\left.\operatorname{CRLB}(\boldsymbol{p})\right|_{\boldsymbol{C}_{\boldsymbol{e}}=\mathcal{I}}}=\sqrt{\frac{1}{2} \operatorname{tr}\left\{\left(J^{T}(\boldsymbol{p}) \boldsymbol{J}(\boldsymbol{p})\right)^{-1}\right\}} .
$$

The GDOP can be influenced by an adequate spatial distribution of the ROs. Given a certain GDOP, the CRLB is only influenced by the variances of the pseudorange errors $\sigma_{e_{b}}^{2}$, which should be as small as possible. Since the pseudoranges $\hat{d}_{b}$ are determined from the ToAs $\hat{\tau}_{b, 1}$, which in turn depend on the estimated sampling phase $\hat{\varepsilon}_{b}$ according to (7), the performance limits for $\mathrm{CPE}$ are required in order to determine a CRLB for positioning:

$$
\sigma_{e_{b}}^{2}=\mathrm{c}^{2} \cdot \sigma_{\tau_{b, 1}}^{2}=\mathrm{c}^{2} \cdot \sigma_{\varepsilon_{b}}^{2}=\mathrm{c}^{2} \cdot \operatorname{CRLB}\left(\varepsilon_{b}\right) .
$$

Hence, the performance limits for CPE directly translate into the performance limits for positioning given a certain GDOP.

\section{Conclusion}

In this article, the positioning part of the joint communication and positioning system recently proposed by the authors is investigated. CPE is the core part of the system proposal, which is based on IDM in combination with PLACE, and positioning via the ToA. Based on the assumption that a priori information about pulse shaping and receive filtering is available, parameters of the physical channel, that are exploited for positioning, are estimated jointly with the channel coefficients of the equivalent discrete-time channel model, which are needed for data detection. There are two equivalent approaches for CPE, which are based on the maximum-likelihood principle: One approach is based on the received samples and the other approach, which is performed in two steps, is based on preliminary channel estimates obtained via standard channel estimation. The latter approach is recommended since it is advantageous from a complexity point of view. Performance limits in terms of CRLBs are determined for a single-path model, different two-path channel models, and several WINNER channel models. The influence of oversampling and different channel characteristics is investigated. It is shown that oversampling provides a performance gain because the channel power as well as the CRLB for symbol-rate sampling depend on the sampling phase, while they are independent of the sampling phase for oversampling. The influence of the channel characteristics in the two-path channels (namely the excess delay, phase offset and power ratio) can be summarized as follows: Dense multipath scenarios with similar or opposite phases and a small power ratio are most challenging. The more challenging it is to estimate the sampling phase, the higher is the gain due to oversampling. Hence, the application of oversampling (with $J=2$ ) is highly recommended in order to improve the positioning accuracy. The relationships observed for the two-path channels are the basis for more complex channel models with an arbitrary number of propagation paths. In this case, the mutual relationship between all paths determines the performance. Consequently, the performance for the WINNER channels can be predicted. A performance prognosis is derived that is met by the simulation results for the WINNER channel models. All results presented in this article are not limited to the proposed system concept but apply for other multiplexing techniques as well.

\section{Endnote}

a The WINNER channel models applied in this contribution differ slightly from those defined in [33]. In [33], the two strongest clusters are divided into three sub-clusters: The first sub-cluster is composed of ten rays and has a zero delay offset, the second sub-cluster consists of six rays and has a delay offset of $5 \mathrm{~ns}$ and the last sub-cluster comprises 
four rays with a delay offset of $10 \mathrm{~ns}$ [33, p. 41]. This division is neglected here: All 20 rays have the same delay, where the original delay is offset by $3.5 \mathrm{~ns}$ (mean offset).

\section{Abbreviations}

AWGN: additive white Gaussian noise; BPSK: binary phase shift keying; CDL: clustered delay line; CRLB: Cramer-Rao lower bound; ENC: encoder; GDOP: geometric dilution of precision; IDM: interleave-division multiplexing; IDMA: interleave-division multiple access; LOS: line of sight; LS: least-squares; ML: maximum-likelihood; ML-IDMA: multi-layer interleave-division multiple access; MS: mobile station; MSE: mean squared error; PLACE: pilot layer aided channel estimation; PNR: pilot-to-noise ratio; RO: reference object; SNR: signal-to-noise ratio; TDM: time-division multiplexing; ToA: time-of-arrival; WINNER: wireless world initiative new radio.

\section{Competing interests}

The authors declare that they have no competing interests.

\section{Acknowledgements}

This study was partly funded by the German Research Foundation (DFG project number $\mathrm{HO} 2226 / 11-1$ )

Received: 22 December 2011 Accepted: 25 July 2012

Published: 21 August 2012

\section{References}

1. R Haeb-Umbach, S Peschke, A novel similarity measure for positioning cellular phones by a comparison with a database of signal power levels. IEEE Trans. Veh. Technol. 56, 368-372 (2007)

2. G Heinrichs, J Winkel, C Drewes, L Maurer, A Springer, R Stuhlberger, C Wicpalek, System considerations for a combined UMTS/GNSS receiver, in Proceedings of 4th Workshop on Positioning, Navigation and Communication (WPNC), Hannover, Germany, 2007, pp. 189-198

3. S Ali, P Nobles, A novel indoor location sensing mechanism for IEEE 802.11 $\mathrm{b} / \mathrm{g}$ wireless LAN, in Proceedings of 4 th Workshop on Positioning, Navigation and Communication (WPNC), Hannover, Germany, 2007, pp. 9-15

4. R Raulefs, S Plass, C Mensing, The WHERE project-combining wireless communications and navigation, in Proceedings of 20th Wireless World Research Forum (WWRF), Ottawa, Canada, 2008, pp. 1-5

5. PA Hoeher, K Schmeink, Joint navigation communication based on interleave-division multiple access, in Proceedings of 6th International Workshop on Multi-Carrier Spread Spectrum (MCSS), Herrsching, Germany, 2007, pp. 97-106

6. K Schmeink, PA Hoeher, Multi-layer interleave-division multiple access for joint communication and localization, in Proceedings of 7 th International ITG-Conference on Source and Channel Coding (SCC), Ulm, Germany, 2008, pp. 1-6

7. PA Hoeher, H Schoeneich, JC Fricke, Multi-layer interleave-division multiple access: theory and practice. Eur. Trans. Telecommun. (ETT). 19(5), 523-536 (2008)

8. H Schoeneich, PA Hoeher, Iterative pilot-layer aided channel estimation with emphasis on interleave-division multiple access systems. EURASIP J. Appl. Signal Process. 2006, 1-15 (2006)

9. S Gezici, A survey on wireless position estimation. Wirel. Personal Commun. (Special Issue on Towards Global and Seamless Personal Navigation). 44(3), 263-282 (2008)

10. RB Langley, Dilution of precision. GPS World. 10(5), 52-59 (1999)

11. M Feder, E Weinstein, Parameter estimation of superimposed signals using the EM algorithm. IEEE Trans. Acoust. Speech Signal Process. 36(4), 477-489 (1988)

12. BH Fleury, M Tschudin, R Heddergott, D Dalhaus, KI Pedersen, Channel parameter estimation in mobile radio environments using the SAGE algorithm. IEEE J. Sel. Areas Commun. 17(3), 434-450 (1999)

13. A Richter, M Landmann, RS Thomae, Maximum likelihood channel parameter estimation from multidimensional channel sounding measurements, in Proceedings of 57 th IEEE Vehicular Technology Conference (VTC Spring), Vol, 2. Jeju, Korea, 2003, pp. 1056-1060
14. A Khayrallah, R Ramesh, G Bottomley, D Koil pillai, Improved channel estimation with side information, in Proceedings of 47th IEEE Vehicular Technology Conference (VTC Spring), Vol. 2. Phoenix, Arizona, USA, 1997, pp. 1049-1053

15. JW Liang, B Ng, JT Chen, A Paulraj, GMSK linearization and structured channel estimate for GSM signals, in Proceedings of IEEE Military Communications Conference (MILCOM), Vol. 2, Monterey, California, USA, 1997, pp. 817-821

16. HN Lee, GJ Pottie, Fast adaptive equalization/diversity combining for time-varying dispersive channels. IEEE Trans. Commun. 46(9), 1146-1162 (1998)

17. G Fock, P Schulz-Rittich, A Schenke, H Meyr, Low complexity high resolution subspace-based delay estimation for DS-CDMA, in Proceedings of IEEE International Conference on Communications (ICC), New York, USA, 2002, pp. 31-35

18. F Antreich, J Nossek, W Utschick, Maximum likelihood delay estimation in a navigation receiver for aeronautical applications. Aerosp. Sci. Technol. 12(3), 256-267 (2008)

19. AJ van der Veen, M Vanderveen, A Paulraj, Joint angle and delay estimation using shift-invariance techniques. IEEE Trans. Signal Process. 46(2), 405-418 (1998)

20. PA Hoeher, P Robertson, E Offer, T Woerz, The soft-output principle-reminiscences and new developments. Eur. Trans. Telecommun. 18(8), 829-835 (2007)

21. K Wendlandt, M Khider, M Angermann, P Robertson, Continuous location and direction estimation with multiple sensors using particle filtering, in Proceedings of International Conference in Multisensor Fusion and Integration for Intelligent Systems (MFI), Heidelberg, Germany, 2006, pp. 92-97

22. P Robertson, M Roeckl, M Angermann, Advances in multi-sensor data fusion for ubiquitous positioning: novel approaches for robust localization and mapping. in Proceeding of VDE Kongress. Leipzig, Germany, 2010

23. H Schoeneich, PA Hoeher, JC Fricke, Adaptive 4G uplink proposal based on interleave-division multiple access, in Proceedings of General Assembly of International Union of Radio Science (URSI GA), New Delhi, India, 2005, paper no. C03.5

24. L Ping, L Liu, K Wu, WK Leung, Interleave-division multiple access. IEEE Trans. Wirel. Commun. 55(4), 938-947 (2006)

25. K Kusume, G Dietl, W Utschick, G Bauch, Performance of interleave division multiple access based on minimum mean square error detection. in Proceedings of IEEE International Conference on Communications (ICC), Glasgow, Scotland, 2007, pp. 2961-2966

26. D Hao, PA Hoeher, Superposition modulation with reliability-based hybrid detection. in Proceedings of 6th International Symposium on Turbo Codes \& Iterative Information Processing (ISTC), Brest, France, 2010, pp. 280-284

27. LL Scharf, Statistical Signal Processing: Detection, Estimation, and Time Series Analysis. chap 6, 2nd edn. (Addison-Wesley, Reading, MA, 1991)

28. SM Kay, Fundamentals of Statistical Signal Processing: Estimation Theory (Prentice-Hall, Upper Saddle River, NJ, 1993)

29. J Kennedy, R Eberhart, Particle swarm optimization. in Proceedings of IEEE International Conference on Neural Networks. Perth, Australia, 1995, pp. 1942-1948

30. D Bratton, J Kennedy, Defining a standard for particle swarm optimization. in Proceedings of IEEE Swarm Intelligence Symposium (SIS), Honolulu, Hawaii, 2007, pp. 120-127

31. K Schmeink, R Block, C Knievel, PA Hoeher, Joint channel and parameter estimation for combined communication and navigation using particle swarm optimization. in Proceedings of 7th Workshop on Positioning, Navigation and Communication (WPNC), Dresden, Germany, 2010, pp. 4-9

32. WH Press, SA Teukolsky, WT Vetterling, BP Flannery, Flannery, Numerical Recipes in C++: The Art of Scientific Computing. 2nd edn. Cambridge University Press, Cambridge, England, 2002

33. P Kyoesti, J Meinilae, L Hentilae, X Zhao, T Jaemsae, C Schneider, M Narandzic, M Milojevic, A Hong, J Ylitalo, VM Holappa, M Alatossava, R Bultitude, Y de Yong, T Rautianinen, IST-4-027756 WINNER II D1. 1.2 V1.2, WINNER II Channel Models, (2008). [http://projects.celtic-initiative.org/ winner+/WINNER2-Deliverables/D1.1.2v1.2.pdf]

34. Understanding GPS: Principles and Applications, chap. 2(ED Kaplan, ed.) Artech House, Boston, MA, 1996

35. G Shen, R Zetik, RS Thomae, Performance comparison of TOA and TDOA based location estimation algorithms in LOS environment, in Proceedings 
of 6th Workshop on Positioning, Navigation and Communication (WPNC), Hannover, Germany, 2008, pp. 71-78

36. AH Sayed, A Tarighat, N Khajenouri, Network-based wireless location: challenges faced in developing techniques for accurate wireless location information. IEEE Signal Process. Mag. 22(4), 24-40 (2005)

37. I Guevenc, CC Chong, F Watanabe, H Inamura, NLOS identification weighted least-squares localization for UWB systems using multipath channel statistics. EURASIP J. Appl, Signal Process. 2008 Article ID 271984, $1-14$ (2008)

doi:10.1186/1687-6180-2012-178

Cite this article as: Schmeink et al:: Performance limits of channel parameter estimation for joint communication and positioning. EURASIP Journal on Advances in Signal Processing 2012 2012:178.

\section{Submit your manuscript to a SpringerOpen ${ }^{\mathcal{O}}$ journal and benefit from:}

- Convenient online submission

- Rigorous peer review

- Immediate publication on acceptance

- Open access: articles freely available online

- High visibility within the field

- Retaining the copyright to your article 